\section{A simple, all-mechanical operant apparatus}

FRANK A. LOGAN and FRANK A. LOGAN III, UNIVERSITY OF NEW MEXICO, Alberquerque, New Mexico 87106

An apparatus is described that utilizes the force exerted by a rat in turning a wheel to rotate reward cups into his presence after passing through a reservoir containing water or ground mash. The response-ratio requirement, force requirement, and amount of reward can be manipulated by simple adjustment or interchange of parts.

Ever since B. F. Skinner introduced the basic concepts of operant conditioning and demonstrated the potentialities of automatic programming and recording equipment, those working in that tradition have been at the forefront of improved instrumentation in experimental psychology. In the process, apparatus design has become increasingly complicated and expensive. This was a natural development as the increasing complexity of the experimental analysis of behavior demanded more sophisticated control. The outcome, however, is that simple, inexpensive devices for the study of operant behavior are not generally available.
The purpose of this note is to describe an apparatus that is inexpensive (cost under \$25), entirely mechanical, essentially foolproof, readily constructable, and yet with sufficient versatility to make it of potential value for some instructional and research purposes. A rearview drawing of the apparatus is shown in Fig. 1.

The manipulandum is a small wheel (A) constructed from two 6-in.-diam plastic discs joined by $1 / 8$-in. stainless-steel rods set on $1 / 2$-in. centers. Similar wheels have been used in the context of aversive control (e.g., Miller, 1948). This wheel is connected by gears (B) to a second shaft from which protrude two rods of $1 / 16$-in. brass, at the ends of which are threaded cups (C) made by countersinking a hole into the end of a short piece of $1 / 8$-in. brass rod. With this arrangement, as a rat turns the wheel, the cups are mechanically rotated into the experimental chamber.

Below the cups is a reservoir (D) that may contain water, ground mash, or any substance that will tend to seek a level. As a cup is rotated through this reservoir, it is filled before it continues on into the rat's presence. A mechanical counter (E) is activated by spokes protruding from the gear just as the cup comes into the appropriate position for the rat to have access to it, thus providing a "magazine"

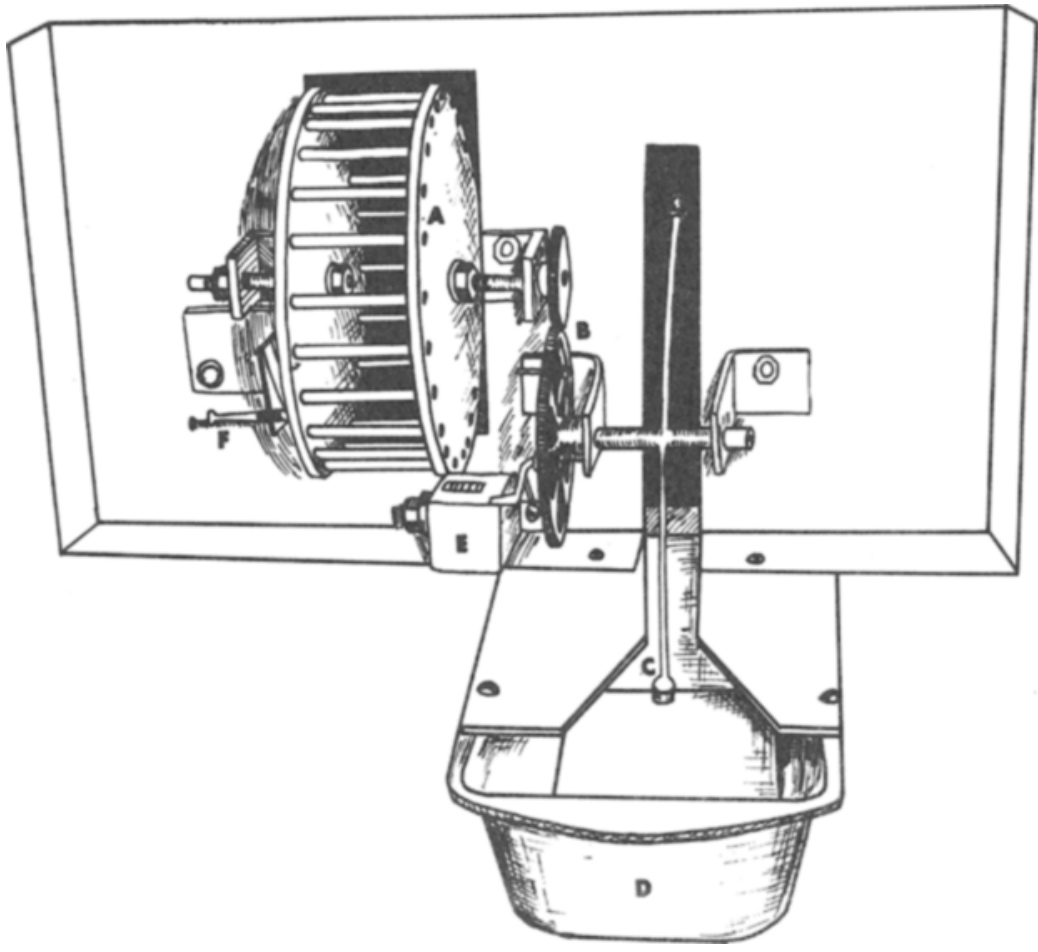

click to serve as a stimulus for the rat to stop turning the wheel.

A spring-loaded resistance (F) presses against the opposite side of the wheel to offer some dampening of the possibility that the cup will coast out of the rat's reach. Further increases in this resistance serve to vary the effort requirement imposed on the rat. By using sets of interchangeable gears, the ratio requirement of wheel turns to rewards can be varied over a moderate range; this range can be increased by changing the number and location of the rotating cups. Finally, the size of the cups can be changed to vary the amount of reward.

Rats readily adapt to this environment. The $\mathrm{E}$ can rotate the wheel from outside the experimental chamber to initiate magazine training and its associated click. Typical shaping procedures follow, although the $\mathrm{E}$ may initially have to help stop the wheel at the appropriate time until the rat learns to anticipate the arrival of the cup. Otherwise, the rat may miss occasional rewards, although they can learn rapidly without any further assistance. In observation, it appears that the wheel-turn response is relatively natural for appetitive responding. Specifically, for example, a rat has earned all of his food and water from two such apparatuses, averaging about 500 revolutions a day on each for over 6 months with no attention except for the periodic refilling of the reservoirs.

\title{
REFERENCE
}

MILLER, N. E. Studies of fear as an acquirable drive: I. Fear as motivation and fear reduction as reinforcement in the learning of new responses. Journal of Experimental Psychology, 1948, 38, 89-101.

Fig. 1. Rearview drawing of the apparatus. The rat turns the wheel (A) from inside the chamber. This response, through gears (B), rotates the cups (C) through a reservoir (D) and on into his presence. A counter (E) is activated by spokes protruding from the larger gear. These are spaced to coincide with the appearance of the cup in the chamber, thus providing a magazine click at the same time rewards are counted. The screw (F) can be tightened to provide resistance against the movement of the wheel. 